\title{
ALMOST SURE GLOBAL WELL-POSEDNESS FOR FRACTIONAL CUBIC SCHRÖDINGER EQUATION ON TORUS
}

\author{
SECKIN DEMIRBAS
}

\begin{abstract}
In [12, we proved that 1-d periodic fractional Schrödinger equation with cubic nonlinearity is locally well-posed in $H^{s}$ for $s>\frac{1-\alpha}{2}$ and globally well-posed for $s>\frac{5 \alpha-1}{6}$. In this paper we define an invariant probability measure $\mu$ on $H^{s}$ for $s<\alpha-\frac{1}{2}$, so that for any $\epsilon>0$ there is a set $\Omega \subset H^{s}$ such that $\mu\left(\Omega^{c}\right)<\epsilon$ and the equation is globally well-posed for initial data in $\Omega$. We see that this fills the gap between the local well-posedness and the global well-posedness range in almost sure sense for $\frac{1-\alpha}{2}<\alpha-\frac{1}{2}$, i.e. $\alpha>\frac{2}{3}$ in almost sure sense.
\end{abstract}

\section{INTRODUCTION}

We consider the cubic periodic fractional Schrödinger equation,

$$
\left\{\begin{array}{l}
i u_{t}+(-\Delta)^{\alpha} u=\gamma|u|^{2} u, \quad x \in[0,2 \pi], \quad t \in \mathbb{R}, \\
u(x, 0)=u_{0}(x) \in H^{s}([0,2 \pi]),
\end{array}\right.
$$

where $\alpha \in(1 / 2,1)$ and $\gamma= \pm 1$. The equation is called focusing for $\gamma=1$ and defocusing for $\gamma=-1$

On real line, this equation arises as a model in the theory of fractional quantum mechanics, see [16]. In [15], Kirkpatrick, Lenzmann and Staffilani derived it as a continuum limit of a model for the interaction of quantum particles on lattice points. Allowing only the nearest points to interact gives the usual cubic Schrödinger equation whereas allowing long range interactions gives rise to the fractional Schrödinger equations with paremeter $\alpha$.

For $\alpha=1$, in [1], Bourgain proved periodic Strichartz's estimates and showed $L^{2}$ local and global well-posedness for the cubic Schrödinger equation. In [6], Burq, Gerard and Tzvetkov noted that this result is sharp since the solution operator is not uniformly continuous on $H^{s}$ for $s<0$.

Date: September 4, 2018. 
The fractional Schrödinger equation on real line was recently studied in [9]. For $\alpha \in$ $(1 / 2,1)$, the equation is less dispersive, so one would not expect to be able to get local well-posedness on $L^{2}$ level. Indeed, they proved that there is local well-posedness on $H^{s}$ for $s \geq \frac{1-\alpha}{2}$. They also showed that the solution operator fails to be uniformly continuous in time for $s<\frac{1-\alpha}{2}$.

In 12, we proved that the periodic fractional equation is locally well-posed in $H^{s}$, for $s>\frac{1-\alpha}{2}$ using direct $X^{s, b}$ estimates. Further, we proved a Strichartz's estimate of the form,

$$
\left\|e^{i t(-\Delta)^{\alpha}} f\right\|_{L_{t \in \mathbb{R}}^{4} L_{x \in \mathbb{T}}^{4}} \lesssim\|f\|_{H^{s}}
$$

for $s>\frac{1-\alpha}{4}$, which also gives local well-posedness for $s>\frac{1-\alpha}{2}$, using the methods in [8] and [11].

Moreover, we proved in [12] that the defocusing equation is globally well-posed for $s>$ $\frac{5 \alpha+1}{6}$, using Bourgain's high-low frequency decomposition introduced in [2]. This method uses the decomposition of the equation into the evolutions of the high and the low frequencies of the initial data. Since the low frequancy part is smooth, its evolution is global due to the conservation of the energy. But the same cannot be said for the high frequancy part. To overcome this problem we showed that the nonlinear evolution of the high frequency part is smoother than the initial data. We should mention that for $\alpha=1$ the smoothing coincides with the smoothing estimate for the NLS that was recently obtained in 13 .

After obtaining these local and global well-posedness results, the natural question that arises is how much we can push the global well-posedness range. For example, the cubic periodic Schrödinger equation $(\alpha=1)$ in 1-d is locally well-posed in $L^{2}$, see [1], and with the mass conservation, we know that the equation is globally well-posed. That is, conservation laws on the local well-posedness level may give rise to global well-posedness. But then, one can ask whether we can show that the equation is globally well-posed whenever it is locally well-posed. Although when there is no conservation laws on the local well-posedness level, it is not trivial that this statement is true, we can still make sense of the question in a different way. The idea relies on the intuition that the set of 'bad' initial data, where the solutions of the equation with those initial data, may have arbitrarily large norm, should be negligible. This approach of looking at the problem in an 'almost sure' sense originated from the work of Lebowitz, Rose and Spear, [17. They were trying to understand the general 
behavior of a system containing a large number of particles by looking at the values of the observables by taking averages over certain probability distributions containing only a few parameters instead of looking at the individual initial value problems. With this in mind, they constructed probability measures on Sobolev spaces and proved some basic properties of these measures.

Later, Bourgain. in 3], proved that the Schrödinger equation with power nonlinearity,

$$
\left\{\begin{array}{l}
i u_{t}-\Delta u=-|u|^{p-2} u, \quad x \in[0,2 \pi], \quad t \in \mathbb{R} \\
u(x, 0)=u_{0}(x) \in H^{s}([0,2 \pi])
\end{array}\right.
$$

where $4<p \leq 6$ is locally well-posed in $H^{s}$ with $s>0$. But for $0<s<1$ there is no conservation law which would easily allow us to extend the local solutions to global ones. He used the idea of Lebowitz, Rose and Spear to construct a probability measure, also known as Gibbs' measure, on $H^{s}$ for $s<\frac{1}{2}$ which is invariant under the solution flow. Then he showed that for any $\epsilon>0$, there is global in time $H^{s}$ norm bounds on the solutions with the initial data in $H^{s}$ up to a set of measure less than $\epsilon$, i.e. the equation is almost surely globally well-posed in $H^{s}$ for $0<s<\frac{1}{2}$.

The idea of Gibbs' measures and almost sure global well-posedness later have been used to prove similar results for different equations by [4, [5], [7, [10], 18], [19], [21, [22, 223] and many others.

Our main result here, is the explicit construction of Gibbs' measure for 1-d fractional periodic cubic Schrödinger equation and the proof of almost sure global well-posedness. More precisely, we define an invariant probability measure $\mu$ on $H^{s}$, for $s<\alpha-\frac{1}{2}$ such that for any $\epsilon>0$ we can find a set $\Omega \subset H^{s}$ satisfying $\mu\left(\Omega^{c}\right)<\epsilon$ and the solution to the equation (2) exists globally for all initial data in $\Omega$.

For that, we are going to truncate the equation (2), and use the idea of invariant measures on finite dimensional Hamiltonian systems. Namely, if we look at the equation,

$$
\left\{\begin{array}{l}
i u_{t}^{N}+(-\Delta)^{\alpha} u^{N}=\gamma P_{N}\left|u^{N}\right|^{2} u^{N}, \\
u^{N}(x, 0)=P_{N} u_{0}(x)
\end{array}\right.
$$


where $P_{N}$ is the projection operator onto the first $N$ frequencies, we see that (3) is a finite dimensional Hamiltonian system, with the Hamiltonian,

$$
H_{N}(u)(t)=\left.\left.\frac{1}{2} \sum_{n \leq N}|| n\right|^{\alpha} \widehat{u_{n}(t)}\right|^{2}-\frac{\gamma}{4} \int_{\mathbb{T}}\left|\sum_{n \leq N} e^{i n x} \widehat{u_{n}(t)}\right|^{4}
$$

By Liouville's theorem, we know that the Lebesgue measure $\prod_{|n| \leq N} d \widehat{u_{n}}$ is invariant under the Hamiltonian flow. Thus, by the conservation of the Hamiltonian and the invariance of the Lebesgue measure under the flow, we see that the finite measure,

$$
d \mu_{N}=e^{-H_{N}(u)} \prod_{|n| \leq N} d \widehat{u_{n}}
$$

is invariant under the solution operator, call it $\mathrm{S}(\mathrm{t})$.

We see that equation (2) is an infinite dimensional Hamiltonian system on the Fourier side with the Hamiltonian,

$$
H(u(t))=\left.\left.\frac{1}{2} \sum_{n}|| n\right|^{\alpha} \widehat{u_{n}(t)}\right|^{2}-\frac{\gamma}{4} \int_{\mathbb{T}}\left|\sum_{n} e^{i n x} \widehat{u_{n}(t)}\right|^{4}=H\left(u_{0}\right),
$$

Then we define the limiting measure $\mu$ on $H^{s}$ as,

$$
d \mu=e^{-H(u)} \prod_{n} d \widehat{u_{n}}=e^{-\left.\left.\frac{1}{2} \sum_{n}|| n\right|^{\alpha} \widehat{u_{n}(t)}\right|^{2}+\frac{\gamma}{4} \int_{\mathbb{T}}\left|\sum_{n} e^{i n x} \widehat{u_{n}(t)}\right|^{4}} \prod_{n} d \widehat{u_{n}},
$$

and show that the measure $\mu$ is indeed the weak limit of $\mu_{N}$.

To construct this measure $\mu$ on appropriate $H^{s}$ spaces, we use the theory of Gaussian measures on Hilbert spaces following Zhidkov's arguments in [24], and first define,

$$
d w=e^{-\left.\left.\frac{1}{2} \sum_{n}|| n\right|^{\alpha} \widehat{u_{n}(t)}\right|^{2}} \prod_{n} d \widehat{u_{n}} .
$$

Then we show that the measure $\mu$ is absolutely continuous with respect to the Gaussian measure $w$ under certain conditions and finish the proof of almost sure global well-posedness by constructing the set $\Omega \subset H^{s}$ as stated above. For the second part we will mainly use Bourgain's arguments in [3].

\section{Notations And Preliminaries}

Recall that for $s \geq 0, H^{s}(\mathbb{T})$ is defined as a subspace of $L^{2}$ via the norm

$$
\|f\|_{H^{s}(\mathbb{T})}:=\sqrt{\sum_{k \in \mathbb{Z}}\langle k\rangle^{2 s}|\widehat{f}(k)|^{2}},
$$


where $\langle k\rangle:=\left(1+k^{2}\right)^{1 / 2}$ and $\widehat{f}(k)=\frac{1}{2 \pi} \int_{0}^{2 \pi} f(x) e^{-i k x} d x$ are the Fourier coefficients of $f$. We use $(\cdot)^{+}$to denote $(\cdot)^{\epsilon}$ for all $\epsilon>0$ with implicit constants depending on $\epsilon$.

We denote the linear propogator of the equation as $e^{-i t(-\Delta)^{\alpha}}$, which is defined on the Fourier side as

$$
\left(e^{-\widehat{i t(-\Delta)^{\alpha}}} f\right)(n)=e^{i t|n|^{2 \alpha}} \widehat{f}(n),
$$

and $|\nabla|^{\alpha}$ is defined as $\left(\widehat{\left.\nabla\right|^{\alpha} f}\right)(n)=|n|^{\alpha} \widehat{f}(n)$.

When we say the equation (2) is locally well-posed in $H^{s}$, we mean that there exist a time $T_{L W P}=T_{L W P}\left(\left\|u_{0}\right\|_{H^{s}}\right)$ such that the solution exists and is unique in $X_{T_{L W P}}^{s, b} \subset$ $C\left(\left[0, T_{L W P}\right), H^{s}\right)$ and depends continuously on the initial data. We say that the the equation is globally well-posed when $T_{L W P}$ can be taken arbitrarily large. Here $X^{s, b}$ denote the Bourgain spaces, and are defined via the restriction in time, of the norm,

$$
\|u\|_{X^{s, b}} \doteq\left\|e^{i t(-\Delta)^{\alpha}} u\right\|_{H_{t}^{b}(\mathbb{R}) H_{x}^{s}(\mathbb{T})}=\left\|\left\langle\tau-|n|^{2 \alpha}\right\rangle^{b}\langle n\rangle^{s} \widehat{u}(n, \tau)\right\|_{L_{\tau}^{2} l_{(m, n)}^{2}},
$$

and $\langle x\rangle=\left(1+|x|^{2}\right)^{1 / 2}$

By Duhamel Principle, we know that the smooth solutions of (2) satisfy the integral equation

$$
u(t, x)=e^{-i t(-\Delta)^{\alpha}} u_{0}(x)+i \gamma \int_{0}^{t} e^{-i(t-\tau)(-\Delta)^{\alpha}}|u|^{2} u(\tau, x) d \tau .
$$

We note that along with the Hamiltonian conservation, the equation enjoys mass conservation, namely,

$$
M(u)(t)=\int_{\mathbb{T}}|u(t, x)|^{2}=M(u)(0) .
$$

\section{Almost Sure Global Well-Posedness}

The main result of this paper is,

Theorem 3.1. For $s<\alpha-\frac{1}{2}$ and $\epsilon>0$, there exists an invariant probability measure $\mu$ on $H^{s}$ such that the equation (2) is globally well-posed for any initial data $u_{0} \in \Omega \subset H^{s}$ such that $\mu\left(\Omega^{c}\right)<\epsilon$ with,

$$
\|u(t)\|_{H^{s}} \lesssim\left(\log \left(\frac{1+|t|}{\epsilon}\right)\right)^{s+}
$$

As we mentioned above, in the proof of this theorem, we first define the finite dimensional measures $\mu_{N}$, which are invariant under the solution operator of the truncated equation (3), 
and we define $\mu$ as the weak limit of these measures. But then we have to show how the equation (2) and the truncated equation (3) are related, namely,

Lemma 3.2. Let $A \in \mathbb{R}$ and $u_{0} \in H^{s}$ be such that $\left\|u_{0}\right\|_{H^{s}}<A$, and assume that the solution, $u_{N}$, of (3) satisfies $\left\|u_{N}(t)\right\|_{H^{s}}<A$ for $t \leq T$. Then the equation (2) is wellposed in $[0, T]$ and moreover, for any $s^{\prime}<s$, we have,

$$
\left\|u(t)-u_{N}(t)\right\|_{H^{s^{\prime}}} \leq e^{C_{1}(1+A)^{C_{2} T}} N^{s^{\prime}-s}
$$

where $C_{1}$ and $C_{2}$ independent of $s$.

In the proof of this lemma, we use,

Lemma 3.3 (see [14]). For $b, b^{\prime}$ such that $0 \leq b+b^{\prime}<1,0 \leq b^{\prime}<1 / 2$, then we have

$$
\left\|\int_{0}^{t} e^{-i(-\Delta)^{\alpha}(t-\tau)} f(\tau) d \tau\right\|_{X_{T}^{s, b}} \lesssim T^{1-b-b^{\prime}}\|f\|_{X_{T}^{s,-b^{\prime}}}
$$

for $T \in[0,1]$.

Proof. (Proof of Lemma (3.2)

$u(t)-u_{N}(t)=e^{-i t(-\Delta)^{\alpha}}\left(u_{0}-P_{N} u_{0}\right)+i \gamma \int_{0}^{t} e^{-i(t-\tau)(-\Delta)^{\alpha}}\left(|u|^{2} u(\tau)-P_{N}\left(\left|u^{N}\right|^{2} u^{N}\right)(\tau)\right) d \tau$,

and, taking the $L^{\infty}\left([0, T] ; H^{s^{\prime}}\right)$ norms of both sides for $b>\frac{1}{2}$, since $X^{s^{\prime}, b} \subset L^{\infty}\left([0, T], H^{s^{\prime}}\right)$ for $b>\frac{1}{2}$, we get,

$$
\begin{aligned}
\left\|u-u_{N}\right\|_{L^{\infty}\left([0, T], H^{s^{\prime}}\right)} & \leq\left\|u_{0}-P_{N} u_{0}\right\|_{H^{s^{\prime}}}+\left\|\int_{0}^{t} e^{-i(t-\tau)(-\Delta)^{\alpha}}\left(|u|^{2} u(\tau)-P_{N}\left(\left|u^{N}\right|^{2} u^{N}\right)(\tau)\right) d \tau\right\|_{X^{s^{\prime}, b}} \\
& \leq\left\|u_{0}-P_{N} u_{0}\right\|_{H^{s^{\prime}}}+\left(T_{L W P}\right)^{1-b-b^{\prime}}\left\||u|^{2} u-P_{N}\left|u^{N}\right|^{2} u^{N}\right\|_{X^{s^{\prime},-b^{\prime}}} \\
& \leq\left(T_{L W P}\right)^{1-b-b^{\prime}}\left(\left\||u|^{2} u-P_{N}\left(|u|^{2} u\right)\right\|_{X^{s^{\prime},-b^{\prime}}}+\left\|P_{N}\left(|u|^{2} u-\left|u^{N}\right|^{2} u^{N}\right)\right\|_{X^{s^{\prime},-b^{\prime}}}\right) \\
& \quad+\left\|u_{0}-u_{0, N}\right\|_{H^{s^{\prime}}} \\
& \leq I+I I+I I I,
\end{aligned}
$$

for $b^{\prime}<\frac{1}{2}$ such that $b+b^{\prime}<1$.

The term III is easier to estimate, 


$$
I I I=\left\|\sum_{|n|>N} e^{i n x} \widehat{\left(u_{0}\right)_{n}}\right\|_{H^{s^{\prime}}} \leq N^{s^{\prime}-s}\left\|u_{0}\right\|_{H^{s}} \leq N^{s^{\prime}-s} A .
$$

For the term $I$, we first observe that $P_{N}\left(|v|^{2} v\right)=|v|^{2} v$ for $v=P_{\frac{N}{3}} u$, from the convolution property of frequency restriction. Then we write,

$$
\begin{aligned}
I & \leq\left\||u|^{2} u-P_{N}\left(|v|^{2} v\right)\right\|_{X^{s^{\prime}, b^{\prime}}}+\left\|P_{N}\left(|v|^{2} v-|u|^{2} u\right)\right\|_{X^{s^{\prime},-b^{\prime}}} \\
& =\left\||u|^{2} u-|v|^{2} v\right\|_{X^{s^{\prime}, b^{\prime}}}+\left\|P_{N}\left(|v|^{2} v-|u|^{2} u\right)\right\|_{X^{s^{\prime},-b^{\prime}}} \\
& =I_{1}+I_{2} \leq 2 I_{1}
\end{aligned}
$$

Estimating term $I_{1}$ using $X^{s, b}$ estimates and local well-posedness theory, see [12], we see that,

$$
\begin{aligned}
I_{1} & \lesssim\left(T_{L W P}\right)^{1-b-b^{\prime}}\left(\|u\|_{X^{s^{\prime}, b}}+\|v\|_{X^{s^{\prime}, b}}\right)^{2}\|u-v\|_{X^{s^{\prime}, b}} \\
& \lesssim\left(T_{L W P}\right)^{1-b-b^{\prime}} A^{2}\left\|u-P_{\frac{N}{3}} u\right\|_{X^{s^{\prime}, b}} \\
& \lesssim\left(T_{L W P}\right)^{1-b-b^{\prime}} A^{2}\left\|u_{0}-P_{\frac{N}{3}} u_{0}\right\|_{H^{s^{\prime}}} \\
& \lesssim\left(T_{L W P}\right)^{1-b-b^{\prime}} A^{3} N^{s^{\prime}-s} .
\end{aligned}
$$

Thus we get,

$$
I \lesssim\left(T_{L W P}\right)^{1-b-b^{\prime}} A^{3} N^{s^{\prime}-s}
$$

Similarly, for the second term we have,

$$
I I \lesssim\left(T_{L W P}\right)^{1-b-b^{\prime}}\left(\|u\|_{X^{s^{\prime}, b}}+\left\|u^{N}\right\|_{X^{s^{\prime}, b}}\right)^{2}\left\|u-u^{N}\right\|_{X^{s^{\prime}, b}} \lesssim\left(T_{L W P}\right)^{1-b-b^{\prime}} A^{2}\left\|u-u^{N}\right\|_{X^{s^{\prime}, b}}
$$

and collecting all the terms, we get,

$$
\begin{aligned}
\left\|u-u_{N}\right\|_{X^{s^{\prime}, b}} \leq & C N^{s^{\prime}-s} A+C_{2}\left(T_{L W P}\right)^{1-b-b^{\prime}} A^{2}\left\|u-u^{N}\right\|_{X^{s^{\prime}, b}} \\
& +C_{1}\left(T_{L W P}\right)^{1-b-b^{\prime}} A^{3} N^{s^{\prime}-s}, \\
\leq & C A N^{s^{\prime}-s}+\frac{1}{2}\left\|u-u^{N}\right\|_{X^{s^{\prime}, b}} \\
\leq & 2 C A N^{s^{\prime}-s} .
\end{aligned}
$$


for $T_{L W P}$ small enough independent of $N, s$ and $s^{\prime}$. Repeating this argument, since the implicit constant $C$ can be taken independent of $T_{L W P}$ and $N$, we see that at any $T_{L W P}$ time the norm at most doubles and thus, at time $T$ we get,

$$
\left\|u-u_{N}\right\|_{H^{s^{\prime}}} \lesssim 2^{\frac{T}{T_{L W P}}} C A N^{s^{\prime}-s} \approx e^{C^{\prime}(1+A)^{\delta} T} A N^{s^{\prime}-s},
$$

which gives the result.

Now, we define a probability measure on $H^{s}$ using the Hamiltonian. For that we will mainly follow Zhidkov's arguments.

3.1. Construction of the Measure on $H^{s}$ : First we will fix the notation that we will use for the rest of the paper. Let $F=(-\Delta)^{\alpha-s}$ on $H^{s}$. We see that $F$ has the orthonormal eigenfunctions $e_{n}=e^{i n x} /\langle n\rangle^{s}$ in $H^{s}$ with the eigenvalues $|n|^{2 \alpha-2 s}$. We also denote $u_{n}=$ $\left(u, e_{n}\right)_{H^{s}}$.

Definition 3.4. A set $M \subset H^{s}$ is called cylindrical if there exists an integer $k \geq 1$ such that,

$$
M=\left\{u \in H^{s}:\left[u_{-k}, \ldots, u_{-2}, u_{-1}, u_{1}, u_{2}, \ldots, u_{k}\right] \in D\right\}
$$

for a Borel set $D \subset \mathbb{R}^{2 k}$.

We denote by $\mathcal{A}$, the algebra containing all such cylindrical sets. Then we define the additive normalized measure $w$ on the algebra $\mathcal{A}$ as follows: For $M \subset \mathcal{A}$, cylindrical,

$$
w(M)=(2 \pi)^{-k} \prod_{|n|=1}^{k}|n|^{\alpha-s} \int_{D} e^{-\frac{1}{2} \sum_{n=1}^{k}|n|^{2 \alpha-2 s}\left|u_{n}\right|^{2}} \prod_{|n|=1}^{k} d u_{n} .
$$

By the definition of the cylindrical sets, we see that the minimal $\sigma$-algebra $\overline{\mathcal{A}}$ containing $\mathcal{A}$ is the Borel $\sigma$-algebra, see 24]. Although the measure is additive by definition, it doesn't necessarily follow that it is countably additive. Indeed,

Theorem 3.5. The Gaussian measure $w$ is countably additive on $\mathcal{A}$ if and only if $\sum_{n}|n|^{2 s-2 \alpha}<\infty$, i.e. $s<\alpha-\frac{1}{2}$.

Proof. (cf. [24]) Let $\sum_{n}|n|^{2 s-2 \alpha}<\infty$. Wel first show that for any $\epsilon>0$, there exists a compact set $K_{\epsilon} \subset H^{s}$ with $w(M)<\epsilon$ for any cylindrical set $M$ such that $M \cap K_{\epsilon}=\emptyset$. 
Let $b_{n}=|n|^{\tilde{\epsilon}}$ such that $a=\sum_{n}|n|^{2 s-2 \alpha+\tilde{\epsilon}}<\infty$. Then for an arbitrary $R>0$ take the cylindrical sets of the form,

$$
M=\left\{u \in H^{s}:\left[u_{-k}, \ldots, u_{-2}, u_{-1}, u_{1}, \ldots, u_{k}\right] \in D, \quad \text { where } \sum_{|n|=1}^{k}|n| \tilde{\epsilon}_{n}^{\tilde{\epsilon}} u_{n}^{2}>R^{2}\right\} .
$$

Then we see that,

$$
\begin{aligned}
w(M) & =(2 \pi)^{-k} \prod_{|n|=1}^{k}|n|^{\alpha-s} \int_{\sum_{n=1}^{k}|n| \tilde{\epsilon} u_{n}^{2}>R^{2}} e^{-\frac{1}{2} \sum_{n=1}^{k}|n|^{2 \alpha-2 s}\left|u_{n}\right|^{2}} \prod_{|n|=1}^{k} d u_{n} \\
& \leq(2 \pi)^{-k} \prod_{|n|=1}^{k}|n|^{\alpha-s} \int_{\mathbb{R}^{n}} \sum_{n=1}^{k}\left(\frac{|n|^{\tilde{\epsilon}}}{R^{2}} u_{n}^{2}\right) e^{-\frac{1}{2} \sum_{n=1}^{k}|n|^{2 \alpha-2 s}\left|u_{n}\right|^{2}} \prod_{|n|=1}^{k} d u_{n} \\
& \leq R^{-2} \sum_{n}|n|^{2 s-2 \alpha+\tilde{\epsilon}} \\
& =a R^{-2}
\end{aligned}
$$

here, to pass to the third line we used integration by parts with $f=\frac{-u_{n}}{|n|^{2 \alpha-2 s}}$ and $d g=$ $-|n|^{2 \alpha-2 s} u_{n} e^{-\frac{1}{2}|n|^{2 \alpha-2 s} u_{n}^{2}} d u_{n}$. Then, for $R>\sqrt{\frac{a}{\epsilon}}$, we have $w(M)<\epsilon$.

Hence, if we take $K_{\epsilon}=\left\{u \in H^{s}:\left.\sum_{n}|n|\right|^{\tilde{\epsilon}} u_{n}^{2} \leq R^{2}\right\}$, we get the desired compact set.

Now let $A_{1} \supset A_{2} \supset \ldots \supset A_{m} \supset \ldots$ be a sequence of cylindrical sets in $H^{s}$ such that $\bigcap_{m=1}^{\infty} A_{m}=\emptyset$. Then for any $\epsilon>0$ there exists closed cylindrical sets $C_{m} \subset A_{m}$ for all $m$ such that $w\left(A_{m} / C_{m}\right)<\epsilon 2^{-m-2}$. Let $D_{m}=\bigcap_{k=1}^{m} C_{k}$. Then $w\left(A_{m} / D_{m}\right) \leq$ $w\left(\bigcup_{k=1}^{m}\left(A_{k} / C_{k}\right)\right)<\epsilon / 2$. Let $E_{m}=D_{m} \cap K_{\epsilon / 2}$, then $E_{m}$ 's are compact with $E_{m} \subset A_{m}$ and $w\left(A_{m} / E_{m}\right)<\epsilon$. Since $\bigcap_{m} A_{m}=\emptyset, \bigcap_{m} E_{m}=\emptyset$, and since $\left(E_{m}\right)$ is a nested sequence of compact sets, we see that $E_{m}=\emptyset$ for all $m>m_{0}$ for some $m_{0} \in \mathbb{N}$.

Hence, $w\left(A_{m}\right)<w\left(E_{m}\right)+\epsilon<\epsilon$, for all $m>m_{0}$. Thus $w\left(A_{m}\right) \rightarrow 0$, i.e. $w$ is countably additive.

For the converse, assume $w$ is countably additive and also $\sum_{n}|n|^{2 s-2 \alpha}=\infty$, i.e. $s \geq \alpha-\frac{1}{2}$. Then consider two cases,

Case 1: $(s \leq \alpha)$. In this case we see that $|n|^{2 s-2 \alpha} \leq 1$ for any $n$. Consider the cylindrical sets of the form,

$$
M_{k}=\left\{u \in H^{s}:\left|\sum_{|n|=1}^{k}\left(u_{n}^{2}\right)-\lambda_{k}\right|<2 \sqrt{\lambda_{k}}\right\},
$$

where $\lambda_{k}=\sum_{|n|=1}^{k}|n|^{2 s-2 \alpha}$. 
Then we have,

$$
\begin{aligned}
w\left(M_{k}^{c}\right) & \left.=w\left(\left\{u \in H^{s}: \mid \sum_{|n|=1}^{k}\left(u_{n}^{2}\right)-\lambda_{k}\right) \mid \geq 2 \sqrt{\lambda_{k}}\right\}\right) \\
& \leq \int_{\mathbb{R}^{2 n}} \frac{\left(\sum_{|n|=1}^{k}\left(u_{n}^{2}\right)-\lambda_{k}\right)^{2}}{4 \lambda_{k}} e^{-\frac{1}{2} \sum_{|n|=1}^{k}|n|^{2 \alpha-2 s}\left|u_{n}\right|^{2}} \prod_{|n|=1}^{k} d u_{n} \\
& =\frac{1}{4 \lambda_{k}} \int_{\mathbb{R}^{2 n}}\left(\left(\sum_{|n|=1}^{k} u_{n}^{2}\right)^{2}-2\left(\sum_{|n|=1}^{k} u_{n}^{2}\right) \lambda_{k}+\lambda_{k}^{2}\right) e^{-\frac{1}{2} \sum_{|n|=1}^{k}|n|^{2 \alpha-2 s}\left|u_{n}\right|^{2}} \prod_{|n|=1}^{k} d u_{n} \\
& =\frac{1}{4 \lambda_{k}}\left(\left(\lambda_{k}^{2}+2 \sum_{|n|=1}^{k}|n|^{4 s-4 \alpha}\right)-2 \lambda_{k} \cdot \lambda_{k}+\lambda_{k}^{2}\right) \\
& \leq \frac{1}{2} \frac{\sum_{|n|=1}^{k}|n|^{4 s-4 \alpha}}{\lambda_{k}} \\
& \leq \frac{1}{2},
\end{aligned}
$$

where, to pass from the third line to the fourth line we used integration by parts again. Since $\lambda_{k} \rightarrow \infty$ as $k \rightarrow \infty$, there exist balls $B_{\lambda_{k}-2 \sqrt{\lambda_{k}}}(0)$ of arbitrarily large radii with $w\left(B_{\lambda_{k}-2 \sqrt{\lambda_{k}}}(0)\right) \leq w\left(M_{k}^{c}\right) \leq \frac{1}{2}$, which contradicts with the countably additivity of $w$.

Case 2: $(s>\alpha)$. In this case, for each $n \geq 1$, consider the cylindrical set

$$
M_{k}=\left\{u \in H^{s}:\left|u_{i}\right| \leq k, \quad|i|=1,2, \ldots, a_{k}\right\},
$$

where $a_{k}>0$ is an integer. Then by a change of variables, we have,

$$
\begin{aligned}
w\left(M_{k}\right) & =(2 \pi)^{-a_{k}} \prod_{|n|=1}^{a_{k}}\left(\int_{-k|n|^{\alpha-s}}^{k|n|^{\alpha-s}} e^{-\frac{1}{2}\left|u_{n}\right|^{2}} d u_{n}\right) \\
& \leq\left[(2 \pi)^{-1} \int_{-k}^{k} e^{-\frac{1}{2}|x|^{2}} d x\right]^{a_{k}},
\end{aligned}
$$

since $s>\alpha$. By choosing $a_{k}$ large enough, we can take $w\left(M_{k}\right) \leq 2^{-k-1}$ for each $k$ and that $a_{k} \rightarrow \infty$ as $k \rightarrow \infty$. Then since $\bigcup_{k=1}^{\infty} M_{k}=H^{s}$ and $w\left(H^{s}\right)=1$, since $H^{s}$ is a cylindrical set with full measure. But then $w\left(\bigcup_{k=1}^{\infty} M_{k}\right) \leq \sum_{k=1}^{\infty} w\left(M_{k}\right) \leq \frac{1}{2}$, which is a contradiction. Hence the theorem follows.

Now we define the sequence of finite dimensional measures $\left(w_{k}\right)$ as follows: For any fixed $k \geq 1$, we take the $\sigma$-algebra, $\mathcal{A}_{n}$, of cylindrical sets in $H^{s}$ of the form $M_{k}=\left\{u \in H^{s}\right.$ : 
$\left.\left[u_{-k}, \ldots, u_{-2}, u_{-1}, u_{1}, \ldots, u_{k}\right] \in D\right\}$, for some Borel set $D \subset \mathbb{R}^{2 k}$. Then,

$$
w_{k}\left(M_{k}\right)=(2 \pi)^{-k} \prod_{|n|=1}^{k}|n|^{\alpha-s} \int_{D} e^{-\frac{1}{2} \sum_{|n|=1}^{k}|n|^{2 \alpha-2 s}\left|u_{n}\right|^{2}} \prod_{|n|=1}^{k} d u_{n} .
$$

Hence we get the sequence of finite dimensional, countably additive measures $w_{k}$ on the $\sigma$-algebra $\mathcal{A}_{k}$. We can also extend these measures to the $\sigma-$ algebra $\overline{\mathcal{A}}$ in $H^{s}$, by setting,

$$
w_{k}(A)=w_{k}\left(A \cap H_{k}^{s}\right), \text { for } A \in \overline{\mathcal{A}},
$$

where $H_{k}^{s}=\operatorname{span}\left(e_{-k}, \ldots, e_{-1}, e_{1}, \ldots, e_{k}\right)$, since $A \cap H_{k}^{s}$ is a Borel subset of $H_{k}^{s}$ for $A \in \overline{\mathcal{A}}$, see $[24$.

The immediate question is whether or not the infinite dimensional Gaussian measure $w$ and the finite measures $w_{k}$ are related and the answer is,

Proposition 3.6. The sequence $w_{k}$ converge weakly to the measure $w$ on $H^{s}$ for $s<\alpha-\frac{1}{2}$ as $k \rightarrow \infty$.

Proof. (cf. [24]) First, recall that a sequence of measures $v_{m}$ is said to converge to a measure $v$ weakly on $H^{s}$ if and only if for any continuous bounded functional $\phi$ on $H^{s}$,

$$
\int \phi(u) d v_{m}(u) \rightarrow \int \phi(u) d v(u)
$$

Also recall that any $\epsilon>0$, if we take $K_{\epsilon} \subset H^{s}$ as in the Theorem (3.5), we see that $w\left(K_{\epsilon}\right)>1-\epsilon$ and moreover, $w_{m}\left(K_{\epsilon}\right)>1-\epsilon$ for all $n \geq 1$. Now let $\phi$ be an arbitrary continuous bounded functional on $H^{s}$ with $B=\sup _{u \in H^{s}} \phi(u)$. Then for any $\epsilon>0$ there exists $\delta=\delta(\epsilon)>0$ such that

$$
|\phi(u)-\phi(v)|<\epsilon \text { for any } u \in K_{\epsilon} \text { and } v \in H^{s} \text { satisfying }\|u-v\|_{H^{s}}<\delta \text {. }
$$

For any $m$, call $K_{m}=K_{\epsilon} \cap H_{m}^{s}$. Then by the definition of the measures $w_{m}$ on $\overline{\mathcal{A}}$, we see that,

$$
\left|\int_{H^{s}} \phi(u) d w_{m}(u)-\int_{K_{m}} \phi(u) d w_{m}(u)\right|<\epsilon B
$$

for any $m \geq 1$. Define,

$$
K_{m, \epsilon}=\left\{v \in H^{s}: v=v_{1}+v_{2}, \quad v_{1} \in H_{m}^{s}, \quad v_{2}^{\perp} \in H_{m}^{s}, \quad\left\|v_{2}\right\|_{H^{s}}<\frac{\delta}{2}, \quad \operatorname{dist}\left(v_{1}, K_{m}\right)<\frac{\delta}{2}\right\} .
$$


Then, $K_{\epsilon} \subset K_{m, \epsilon}$ for all sufficiently large $m$ 's. Thus, for $m$ large enough,

$$
\left|\int_{H^{s}} \phi(u) d w_{m}(u)-\int_{K_{m, \epsilon}} \phi(u) d w_{m}(u)\right|<\epsilon B .
$$

We now define the measure $w_{m}^{\perp}$ on $\left(H_{m}^{s}\right)^{\perp}$ as follows:

For a cylindrical set

$$
M^{\perp}=\left\{u \in\left(H_{m}^{s}\right)^{\perp}:\left[u_{-m-k}, \ldots, u_{-m-2}, u_{-m-1}, u_{m+1}, u_{m+2}, \ldots, u_{m+k}\right] \in F\right\},
$$

where $F \subset \mathbb{R}^{2 k}$ is a Borel set, and,

$$
w_{m}^{\perp}\left(M^{\perp}\right)=(2 \pi)^{-k} \prod_{|n|=m+1}^{m+k}|n|^{\alpha-s} \int_{F} e^{-\frac{1}{2} \sum_{|n|=m+1}^{m+k}|n|^{2 \alpha-2 s}\left|u_{n}\right|^{2}} \prod_{|n|=m+1}^{m+k} d u_{n} .
$$

then we see that $w_{m}^{\perp}$ is a probability measure on $\left(H_{m}^{s}\right)^{\perp}$ and $w=w_{m} \otimes w_{m}^{\perp}$.

Thus we get,

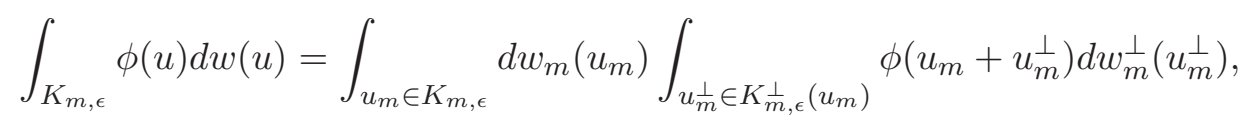

where, $K_{m, \epsilon}^{\perp}\left(u_{m}\right)=K_{m, \epsilon} \cap\left\{u \in H^{s}: u=u_{m}+y, y \in\left(H_{m}^{s}\right)^{\perp}\right\}$. Then by ([6)),

$$
\begin{aligned}
\int_{K_{m, \epsilon}} \phi(u) d w(u) & \left.=\int_{u_{m} \in K_{m, \epsilon}} d w_{m}\left(u_{m}\right) \int_{u_{m}^{\perp} \in K_{m}^{\perp}, \epsilon}\left(\phi\left(u_{m}\right)+u_{m}^{\perp}\right)-\phi\left(u_{m}\right)\right)+\phi\left(u_{m}\right) d w_{m}^{\perp}\left(u_{m}^{\perp}\right) \\
& \leq C \epsilon+\int_{u_{m} \in K_{m, \epsilon}} \phi\left(u_{m}\right) d w_{m}\left(u_{m}\right)
\end{aligned}
$$

for $C$ independent of $m$ and $\epsilon$.

Hence,

$$
\int_{K_{m, \epsilon}} \phi(u) d w(u)-\int_{u_{m} \in K_{m, \epsilon}} \phi\left(u_{m}\right) d w_{m}\left(u_{m}\right) \leq C \epsilon .
$$

Therefore, combining (7), (8) and (10), we get the result.

Now, we show that the measure $\mu$ is absolutely continuous with respect to the Gaussian measure $w$. Recall that,

$$
\begin{aligned}
d \mu_{N} & =(2 \pi)^{-N} \prod_{|n|=1}^{N}|n|^{\alpha-s} e^{-\left.\left.\frac{1}{2} \sum_{|n| \leq N}|| n\right|^{\alpha-s} u_{n}(t)\right|^{2}-\frac{\gamma}{4} \int_{\mathbb{T}}\left|\sum_{|n| \leq N} \frac{e^{i n x}}{\langle n\rangle^{s}} u_{n}(t)\right|^{4}} d u_{0} \prod_{1 \leq|n| \leq N} d u_{n} \\
& =e^{-\frac{\gamma}{4} \int_{\mathbb{T}}\left|\sum_{|n| \leq N} \frac{e^{i n x}}{\langle n\rangle^{s}} u_{n}(t)\right|^{4}}(2 \pi)^{-N} \prod_{|n|=1}^{N}|n|^{\alpha-s} e^{-\frac{1}{2} \sum_{0<|n| \leq N}|n|^{2 \alpha-2 s}\left|u_{n}(t)\right|^{2}} d u_{0} \prod_{1 \leq|n| \leq N} d u_{n},
\end{aligned}
$$

and thus, $\mu_{N}$ is a weighted Gaussian measure. 
For the defocusing NLS, since

$$
\left|u_{0}\right|^{2} \leq \int_{\mathbb{T}}|u|^{2} \lesssim\left(\int_{\mathbb{T}}|u(t)|^{4}\right)^{\frac{1}{2}}
$$

we have,

$$
\int_{u_{0} \in \mathbb{C}} e^{-\frac{1}{4} \int_{\mathbb{T}}\left|\sum_{n} \frac{e^{i n x}}{\langle n\rangle^{5}} u_{n}(t)\right|^{4}} d u_{0} \lesssim \int_{u_{0} \in \mathbb{C}} e^{-\frac{1}{4}\left|u_{0}\right|^{4}} d u_{0} \lesssim C
$$

uniformly in $N$. Thus, instead of working with the full measure $\mu_{N}$ it is enough to work with the measure $w_{N}$, which is also known as the Wiener measure.

For the focusing NLS, though, we don't have an a priori control over the weight $e^{\frac{1}{4} \int_{\mathbb{T}}\left|\sum_{n \leq N} e^{i n x} \widehat{u_{n}(t)}\right|^{4}}$. We can overcome this problem by using a lemma of Lebovitz et al., see[17], which applies an $L^{2}$ cut-off to the set of initial data, namely,

Lemma 3.7. $e^{\frac{1}{4} \int\left|\sum_{1 \leq|n| \leq N} e^{i n x} \widehat{u_{n}(t)}\right|^{4}} \chi_{\left\{\|u\|_{L^{2}} \leq B\right\}} \in L^{1}\left(d w_{N}\right)$ uniformly in $N$, for all $B<\infty$.

Proof. (cf. [22])

$$
\begin{aligned}
& \int e^{\frac{1}{4} \int\left|\sum_{1 \leq|n| \leq N} e^{i n x} \widehat{u_{n}(t)}\right|^{4}} \chi_{\left\{\|u\|_{L^{2}} \leq B\right\}} d w=\quad \quad \quad e^{\frac{1}{4} \int\left|\sum_{1 \leq|n| \leq N} e^{i n x} \widehat{u_{n}(t)}\right|^{4}} \chi_{\left\{\|u\|_{L^{2}} \leq B\right\}} d w \\
& \left(\int\left|\sum_{|n|=1}^{N} e^{i n x} \widehat{u_{n}(t)}\right|^{4} \leq K\right) \\
& +\sum_{i=0}^{\infty} \quad \int \quad e^{\frac{1}{4} \int\left|\sum_{1 \leq|n| \leq N} e^{i n x} \widehat{u_{n}(t)}\right|^{4}} \chi_{\left\{\|u\|_{L^{2}} \leq B\right\}} d w \\
& \left(\int\left|\sum_{|n|=1}^{N} e^{i n x} \widehat{u_{n}(t)}\right|^{4} \in\left(2^{i} K, 2^{i+1} K\right]\right) \\
& \leq e^{\frac{1}{4} K^{4}}+\sum_{i=0}^{\infty} e^{\frac{1}{4}\left(2^{i+1} K\right)^{4}} w\left(\left\{\left(\int\left|\sum_{|n|=1}^{N} e^{i n x} \widehat{u_{n}(t)}\right|^{4}>2^{i} K,\|u\|_{L^{2}}<B\right\}\right) .\right.
\end{aligned}
$$

Now to estimate the second term on the right hand side, choose $N_{0}$ dyadic, to be specified later. Now call $N_{i}=N_{0} \cdot 2^{i}$ and let $a_{i}$ be such that $\sum_{i} a_{i}=\frac{1}{2}$.

Then,

$$
w\left(\left\{\left(\|u\|_{L^{4}}>K,\|u\|_{L^{2}}<B\right\}\right) \leq \sum_{i=1}^{\infty} w\left(\left\{\left(\left\|P_{\left\{|n| \approx N_{i}\right\}} u\right\|_{L^{4}}>a_{i} K\right\}\right),\right.\right.
$$

and since by Sobolev embedding we have,

$$
\left\|P_{\left\{|n| \approx N_{i}\right\}} u\right\|_{L^{4}} \lesssim N_{i}^{\frac{1}{4}}\left\|P_{\left\{|n| \approx N_{i}\right\}} u\right\|_{L^{2}}
$$


we see that,

$$
\begin{aligned}
w\left(\left\{\left(\|u\|_{L^{4}}>K,\|u\|_{L^{2}}<B\right\}\right)\right. & \leq \sum_{i=1}^{\infty} w\left(\left\{\left\|P_{\left\{|n| \approx N_{i}\right\}} u\right\|_{L^{4}}>a_{i} K\right\}\right) \\
& \leq \sum_{i=1}^{\infty} w\left(\left\{\left\|P_{\left\{|n| \approx N_{i}\right\}} u\right\|_{L^{2}} \gtrsim a_{i} N_{i}^{-\frac{1}{4}} K\right\}\right) .
\end{aligned}
$$

Letting $a_{i}=C N_{0}^{\epsilon} N_{i}^{-\epsilon}$ and $N_{0}$ such that $K \approx N_{0}^{\frac{1}{4}} B$, i.e. $N_{0} \approx K^{4} B^{-4}$, we get,

$$
\begin{aligned}
w\left(\left\{\left(\|u\|_{L^{4}}>K,\|u\|_{L^{2}}<B\right\}\right)\right. & \leq \sum_{i=1}^{\infty} w\left(\left\{\left(\sum_{|n| \approx N_{i}}\left|\widehat{u_{n}}\right|^{2}\right)^{\frac{1}{2}} \gtrsim a_{i} N_{i}^{-\frac{1}{4}} K\right\}\right) \\
& \approx \sum_{i=1}^{\infty} w\left(\left\{\left(\sum_{|n| \approx N_{i}}\left|u_{n}\right|^{2}\right)^{\frac{1}{2}} \gtrsim a_{i} N_{i}^{-\frac{1}{4}+s} K\right\}\right),
\end{aligned}
$$

and by the estimation of the tail of the Gaussian measure, (cf. (13)), we have,

$$
\begin{aligned}
w\left(\left\{\left(\|u\|_{L^{4}}>K,\|u\|_{L^{2}}<B\right\}\right)\right. & \lesssim \sum_{i=1}^{\infty} e^{-\frac{1}{4} a_{i}^{2} N_{i}^{(2 \alpha-2 s)+2 s-\frac{1}{2}} K^{2}} \\
& \leq \sum_{i=1}^{\infty} e^{-\frac{1}{4} N_{0}^{2 \epsilon} N_{i}^{2 \alpha-\frac{1}{2}-2 \epsilon} K^{2}} \\
& \leq \sum_{i=1}^{\infty} e^{-\frac{1}{4} N_{0}^{2 \alpha-\frac{1}{2}} 2^{\left(2 \alpha-\frac{1}{2}-2 \epsilon\right) i} K^{2}} \\
& \leq e^{-\frac{1}{4}} K^{2} N_{0}^{2 \alpha-\frac{1}{2}} \\
& \approx e^{-\frac{1}{4} K^{2+4\left(2 \alpha-\frac{1}{2}\right)}} B^{4-8 s} .
\end{aligned}
$$

and collecting terms, we obtain,

$$
\begin{aligned}
\int e^{\frac{1}{4} \int|u|^{4}} \chi_{\left\{\|u\|_{L^{2}} \leq B\right\}} d w & \leq e^{\frac{1}{4} K^{4}}+\sum_{i=0}^{\infty} e^{\frac{1}{4}\left(2^{i+1} K\right)^{4}} w\left(\left\{\left(\|u\|_{L^{4}}>2^{i} K,\|u\|_{L^{2}}<B\right\}\right)\right. \\
& \leq e^{\frac{1}{4} K^{4}}+\sum_{i=0}^{\infty} e^{\frac{1}{4}\left(2^{i+1} K\right)^{4}} e^{-\frac{1}{4}\left(2^{i} K\right)^{2+4\left(2 \alpha-\frac{1}{2}\right)}} B^{4-8 \alpha} \\
& <\infty
\end{aligned}
$$

which proves the lemma.

Moreover, observe that for $\|u\|_{L^{2}}<B$, we get $\left|u_{0}\right|^{2} \leq \sum_{n} \frac{\left|u_{n}\right|^{2}}{\langle n\rangle^{2 s}} \leq B^{2}$. Hence, $L^{2}$ cut off also restricts $u_{0}$ to the ball $\left\{u_{0} \in \mathbb{C}:\left|u_{0}\right| \leq B\right\}$, uniformly in $N$. Therefore, combining 
these two results, we get that the measure $\mu_{N}$ is a weighted Gaussian measure with weight being uniformly in $L^{1}$ with respect to the Gaussian measure.

By the construction of the Gaussian measure, we see that for any compact set $E \subset H^{s}$, we have,

$$
w_{N}\left(E \cap H_{N}^{s}\right) \rightarrow w(E) .
$$

Thus, using the result above we get,

$$
\mu_{N}\left(E \cap H_{N}^{s}\right) \rightarrow \mu(E) .
$$

Proof. (Proof of Theorem (3.1)) For the proof of the theorem and the invariance of the measure $\mu$, we follow Bourgain's arguments in [3]. First, for any $\epsilon$ we will construct the sets $\Omega_{N} \subset H^{s}$ such that $\mu_{N}\left(\Omega_{N}^{c}\right)<\epsilon$ and,

$$
\left\|u^{N}(t)\right\|_{H^{s}} \lesssim\left(\log \left(\frac{1+|t|}{\epsilon}\right)\right)^{\frac{1}{2}} .
$$

For that, we fix a large time $T$ and let $\left[-T_{L W P}, T_{L W P}\right]$ be the local well-posedness interval for the equation (2). Then consider the set

$$
\Omega^{K}=\left\{u \in H_{N}^{s}:\|u\|_{H^{s}} \leq K\right\},
$$

where, again, $H_{N}^{s}=\operatorname{span}\left\{e_{n}:|n| \leq N\right\}$. We see that,

$$
\begin{aligned}
w_{N}\left(\left(\Omega^{K}\right)^{c}\right) & =(2 \pi)^{-\frac{N}{2}} \prod_{|n|=1}^{N}|n|^{\alpha-s} \int_{\left\{u \in H_{N}^{s}:\|u\|_{\left.H^{s}>K\right\}}\right.} e^{-\frac{1}{2} \sum_{|n|=1}^{N}|n|^{2 \alpha-2 s}\left|u_{n}\right|^{2}} \prod_{|n|=1}^{N} d u_{n} . \\
& =(2 \pi)^{-\frac{N}{2}} \prod_{|n|=1}^{N}|n|^{\alpha-s} \int_{\left\{u \in H_{N}^{s}: \sum_{|n| \leq N}\left|u_{n}\right|^{2}>K^{2}\right\}} e^{-\frac{1}{2} \sum_{|n|=1}^{N}|n|^{2 \alpha-2 s}\left|u_{n}\right|^{2}} \prod_{|n|=1}^{N} d u_{n} . \\
& =(2 \pi)^{-\frac{N}{2}} e^{-\frac{1}{2} \sum_{|n|=1}^{N}\left|v_{n}\right|^{2}} \prod_{|n|=1}^{N} d v_{n} . \\
& \leq(2 \pi)^{-\frac{N}{2}} \int_{\left\{\sum_{|n| \leq N} \frac{\left|v_{n}\right|^{2}}{\left.\langle n\rangle^{2 \alpha-2 s}>K^{2}\right\}}\right.} \int_{|n| \leq N}^{v_{\left.\left|v_{n}\right|^{2}>K^{2}\right\}}} e^{-\frac{1}{2} \sum_{|n|=1}^{N}\left|v_{n}\right|^{2}} \prod_{|n|=1}^{N} d v_{n} \\
& =(2 \pi)^{-\frac{N}{2}} \int_{S_{2 N}}^{\infty} \int_{K}^{2 N-1} r^{-\frac{1}{2} r^{2}} d r d S_{2 N}
\end{aligned}
$$




$$
\begin{aligned}
& =(2 \pi)^{-\frac{N}{2}} \int_{S_{2 N}} \int_{K}^{\infty} r \underbrace{r^{2 N-2} e^{-\epsilon(r-\epsilon)-\frac{1}{2} \epsilon^{2}}}_{\leq C} e^{-\frac{1}{2}(r-\epsilon)^{2}} d r d S_{2 N} \\
& \lesssim(2 \pi)^{-\frac{N}{2}} \int_{S_{2 N}} \int_{K}^{\infty} r e^{-\frac{1}{2}(r-\epsilon)^{2}} d r d S_{2 N} \\
& \lesssim(2 \pi)^{-\frac{N}{2}} \int_{S_{2 N}} \int_{K}^{\infty}(r-\epsilon) e^{-\frac{1}{2}(r-\epsilon)^{2}} d r d S_{2 N} \\
& \leq e^{-\frac{1}{2}(K-\epsilon)^{2}} \lesssim e^{-\frac{1}{4} K^{2}},
\end{aligned}
$$

for $\epsilon$ small enough. Thus, $\mu_{N}\left(\left(\Omega^{K}\right)^{c}\right) \lesssim e^{-\frac{1}{4} K^{2}}$.

Since $\mu_{N}$ is invariant under the solution operator, $S_{N}$ of the truncated equation, if we define the set,

$$
\Omega_{N}^{\prime}=\Omega^{K} \cap S_{N}^{-1}\left(\Omega^{K}\right) \cap S_{N}^{-2}\left(\Omega^{K}\right) \cap \ldots \cap S_{N}^{-\frac{T}{T_{L W P}}}\left(\Omega^{K}\right)
$$

$\Omega_{N}^{\prime}$ satisfies the property, $\mu_{N}\left(\left(\Omega_{N}^{\prime}\right)^{c}\right) \leq \frac{T}{T_{L W P}} \mu_{N}\left(\left(\Omega^{K}\right)^{c}\right)<T K^{\theta} e^{-\frac{1}{4} K^{2}}$, since the local wellposedness interval $\left[-T_{L W P}, T_{L W P}\right]$ depends uniformly on the $H^{s}$ norm of the initial data. Thus if we pick $K=\left((4+2 \theta) \log \left(\frac{T}{\epsilon}\right)\right)^{\frac{1}{2}}$, for $\epsilon$ small we get,

$$
\mu_{N}\left(\left(\Omega_{N}^{\prime}\right)^{c}\right)<\epsilon
$$

and by the construction of the set $\Omega_{N}^{\prime}$ we have,

$$
\left\|u^{N}(t)\right\|_{H^{s}} \lesssim\left(\log \left(\frac{T}{\epsilon}\right)\right)^{\frac{1}{2}}
$$

for all $|t|<T$. Moreover, if we take $T_{j}=2^{j}$ and $\epsilon_{j}=\frac{\epsilon}{2^{j+1}}$, and construct $\Omega_{N, j}$ 's, we see that $\Omega_{N}=\bigcap_{j=1}^{\infty} \Omega_{N, j}$, satisfies (12).

Also by the approximation lemma (5), we see that for any $s^{\prime}<s$ we have,

$$
\|u(t)\|_{H^{s^{\prime}}}<2 A \leq C_{s^{\prime}}\left(\log \left(\frac{T}{\epsilon}\right)\right)^{\frac{1}{2}} .
$$

Again by taking an increasing sequence of times, we get,

$$
\|u(t)\|_{H^{s^{\prime}}} \leq C_{s^{\prime}}\left(\log \left(\frac{1+|t|}{\epsilon}\right)\right)^{\frac{1}{2}} .
$$

Hence, if we intersect this result with an increasing sequence of $s<\alpha-\frac{1}{2}$, and taking $\Omega=\bigcap_{N} \Omega_{N}$ where $\left(\Omega_{N}\right)$ s are defined as above with $\mu_{N}\left(\Omega_{N}^{c}\right)<\frac{\epsilon}{2^{N}}$, we get that $\mu(\Omega)<\epsilon$ and 
that the solutions to the equation (2) has the norm growth bound,

$$
\|u(t)\|_{H^{s}} \leq C_{s}\left(\log \left(\frac{1+|t|}{\epsilon}\right)\right)^{\frac{1}{2}}
$$

for initial data $u_{0} \in \Omega$. Moreover, interpolating this bound with,

$$
\|u(t)\|_{L^{2}}=\left\|u_{0}\right\|_{L^{2}}
$$

we have,

$$
\|u(t)\|_{H^{s}} \leq C\left(\log \left(\frac{1+|t|}{\epsilon}\right)\right)^{s+}
$$

which proves theorem (3.1).

3.2. Invariance of $\mu$ Under the Solution Flow. Let $K$ be a compact set and $B_{\epsilon}$ denote the $\epsilon$ ball in $H^{s}$. Let $S$ be the flow map for the equation (2) and $S_{N}$ be the flow map for the equation (3). Then by the weak convergence of the measure,

$$
\mu\left(S(K)+B_{\epsilon}\right)=\lim _{N \rightarrow \infty} \mu_{N}\left(\left(S(K)+B_{\epsilon}\right) \cap H_{N}^{s}\right) .
$$

Also by the uniform convergence of the solutions of (3) to (2) in $H^{s_{1}}$ for any $s_{1}<s$, we get,

$$
S_{N}\left(P_{N} K\right) \subset S(K)+B_{\epsilon / 2}
$$

for $N \geq N_{0}$ sufficiently large. Then for $\epsilon_{1}$ small enough,

$$
S_{N}\left(\left(K+B_{\epsilon}\right) \cap H_{N}^{s}\right) \subset S_{N}\left(P_{N} K\right)+B_{\epsilon / 2} \subset S(K)+B_{\epsilon} .
$$

Hence,

$$
\mu_{N}\left(S_{N}\left(\left(K+B_{\epsilon_{1}}\right) \cap H_{N}^{s}\right)\right) \leq \mu_{N}\left(S(K)+B_{\epsilon}\right),
$$

and by the invariance of $\mu_{N}$, we get,

$$
\mu_{N}\left(\left(K+B_{\epsilon_{1}}\right) \cap H_{N}^{s}\right) \leq \mu_{N}\left(S(K)+B_{\epsilon}\right),
$$

and letting $N \rightarrow \infty$, by the convergence of the measures $\mu_{N}$ to $\mu$,

$$
\mu(K) \leq \mu\left(K+B_{\epsilon_{1}}\right) \leq \mu\left(S(K)+B_{\epsilon}\right),
$$

which say, by the arbitrariness of $\epsilon$, that $\mu(K) \leq \mu(S(K))$, and by the time reversibility, we also have the inverse inequality and, thus,

$$
\mu(K)=\mu(S(K)),
$$


which gives the invariance of $\mu$ under the solution operator.

Acknowledgement: The author wants to thank Burak Erdoğan and Nikolaos Tzirakis for their valuable comments and support.

\section{REFERENCES}

[1] J. Bourgain, Fourier transform restriction phenomena for certain lattice subsets and applications to nonlinear evolution equations, Geometric and Functional Analysis, Vol. 3 (1993), 107-156.

[2] J. Bourgain, Refinements of Strichartz' inequality and applications to 2-D NLS with critical nonlinearity, Int. Math. Res. Not., 5 (1998), 253-283.

[3] J. Bourgain, Periodic nonlinear Schrödinger equation and invariant measures, Comm. Math. Phys. 166 (1994), no. 1, 1-26.

[4] J. Bourgain, A. Bulut, Gibbs measure evolution in radial nonlinear wave and Schrödinger equations on the ball, C. R. Math. Acad. Sci. Paris 350 (2012), no. 11-12, 571-575.

[5] J. Bourgain, A. Bulut, Invariant Gibbs measure evolution for the radial nonlinear wave equation on the 3d ball, J. Funct. Anal. 266 (2014), no. 4, 2319-2340.

[6] N. Burq, P. Gerard, N. Tzvetkov, An instability property of the nonlinear Schrödinger equation on $S^{d}$, Math. Res. Let. 9 (2002), 323-335.

[7] N. Burq, N. Tzvetkov, Invariant measure for a three dimensional nonlinear wave equation, Int. Math. Res. Not. IMRN 2007, no. 22, Art. ID rnm108, 26 pp.

[8] F. Catoire, and W-M. Wang, Bounds on Sobolev norms for the nonlinear Schrödinger equation on general tori, Communications in Pure and Applied Analysis, Vol. 9, 2 (2010), 483-491.

[9] Y. Cho, G. Hwang, S. Kwon, S. Lee, Well-posedness and Ill-posedness for the cubic fractional Schrödinger equations, preprint arXiv:1311.0082

[10] J. Colliander, T. Oh, Almost sure well-posedness of the cubic nonlinear Schrödinger equation below $L^{2}(\mathbb{T})$. Duke Math. J., 161, (2012), no. 3, 367-414.

[11] S. Demirbas, Local Well-posedness for 2-D Schrödinger Equation on Irrational Tori and Bounds on Sobolev Norms, preprint arXiv:1307.0051.

[12] S. Demirbas, M. B. Erdogan, and N. Tzirakis, Existence and Uniqueness theory for the fractional Schrödinger equation on the torus, preprint arXiv:1312.5249

[13] M. B. Erdogan, and N. Tzirakis, Talbot effect for the cubic nonlinear Schrödinger equation on the torus, to appear in Math. Res. Let., preprint arXiv:1303.3604

[14] J. Ginibre, Le problème de Cauchy pour des EDP semi-linéaires périodiques en variables d'espace, Inventiones Mathematicae, Vol. 37, (1995), 163-187.

[15] K. Kirkpatric, E. Lenzmann, G. Staffilani, On the continuum limit for discrete NLS with long-range lattice interactions, Comm. Math. Phys., 317:3 (2013), 563-591. 
[16] N, Laskin, Fractional quantum mechanics and Lévy path integrals, Phys. Lett. A, 268 (2000), 298-305.

[17] J. L. Lebowitz, H. A. Rose, E. R. Speer, Statistical mechanics of the nonlinear Schrödinger equation, vol. 50, issue 3-4 (1988), 657-687.

[18] A. R. Nahmod, T. Oh, G. Staffilani, Invariant weighted Wiener measures and almost sure global wellposedness for the periodic derivative NLS, J. Eur. Math. Soc. (JEMS), 14, (2012), no. 4, 1275-1330.

[19] A. R. Nahmod, N. Pavlović, G. Staffilani, Almost Sure Existence of Global Weak Solutions for Supercritical Navier-Stokes Equations, SIAM J. Math. Anal., 45(6), 3431-3452.

[20] T. Oh, Lecture notes, http://www.maths.ed.ac.uk/ toh/Files/2008_11_27_Hiro_Oh_Gibbs_Measure_Lectures.pdf.

[21] T. Oh, Invariant Gibbs measures and a.s. global well posedness for coupled KdV systems, Differential Integral Equations, 22, (2009), no. 7-8, 637-668.

[22] T. Oh, Invariance of the Gibbs measure for the Schrödinger-Benjamin-Ono system, SIAM J. Math. Anal., 41, (2009/10), no. 6, 2207-2225.

[23] G. Richards, Statistical mechanics of the nonlinear Schrödinger equation, preprint arXiv:1209.4337.

[24] P. Zhidkov, Invariance of the Gibbs measure for the periodic quartic gKdV, No. 1756, Springer, 2001.

Department of Mathematics, University of Illinois, Urbana, IL 61801, U.S.A.

E-mail address: demirba2@illinois.edu 